\title{
DeltaDou: Expert-level Doudizhu AI through Self-play
}

\author{
Qiqi Jiang, Kuangzheng Li, Boyao Du, Hao Chen and Hai Fang \\ SweetCode Inc, Beijing \\ \{jiangqiqi, likuangzheng, duboyao, chenhao, fanghai\}@itgwn.com
}

\begin{abstract}
Artificial Intelligence has seen several breakthroughs in two-player perfect information game. Nevertheless, Doudizhu, a three-player imperfect information game, is still quite challenging. In this paper, we present a Doudizhu AI by applying deep reinforcement learning from games of self-play. The algorithm combines an asymmetric MCTS on nodes representing each player's information set, a policy-value network that approximates the policy and value on each decision node, and inference on unobserved hands of other players by given policy. Our results show that self-play can significantly improve the performance of our agent in this multiagent imperfect information game. Even starting with a weak AI, our agent can achieve human expert level after days of self-play and training.
\end{abstract}

\section{Introduction}

Computer programs are able to beat the best human players in a lot of popular perfect information games, such as backgammon [Tesauro, 1995], checkers [Schaeffer et al., 2007], chess [Campbell et al., 2002], and go [Silver et al., 2016]. AlphaZero [Silver et al., 2018] introduces a general reinforcement learning framework, which is able to master chess, go, and shogi without human knowledge via self-play.

Imperfect information games, where players cannot observe the full state of game, is still very challenging. DeepStack [Moravčík et al., 2017] and Libratus [Brown and Sandholm, 2018] achieve super-human level in Heads-Up No-Limit Texas Hold'em using Counterfactual Regret Minimization (CFR). CFR relies on a complete traversal of the game tree, and the traversal would be difficult for some games with large state space.

In this paper, we describe how to create an expert-level AI player for the game of Doudizhu by integrating two techniques into an AlphaZero-like self-play framework.

The first technique is an asymmetric Monte Carlo Tree Search (MCTS) on information set for each player, called Fictitious Play MCTS (FPMCTS). To alleviate state explosion problem, we construct a game tree of information set nodes for each player. The acting player chooses his action in a PUCT [Silver et al., 2016] fashion, and the other two players' actions are sampled from their own policy networks. This approach avoids the issues of Strategy Fusion and NonLocality. Moreover, FPMCTS converges very fast, since the branching factor is the size of the action space instead of the size of possible determinizations.

The second technique is a policy-based inference algorithm. [Buro et al., 2009] proposes a good inference algorithm that improves performance of their Skat AI drastically. We developed our own inference algorithm in our self-play framework, and found that policy network is a better fit than hand-crafted features in our case.

FPMCTS and the inference algorithm are integrated into a self-play framework. Each self-play episode takes all players' neural networks as input. The search phase returns a vector of probability distribution over moves, and the resulting distribution along with the game results are then used to update the neural network of each player. In the next episode, this updated neural network used in inference and looking-forward search. To bootstrap the reinforcement learning procedure, we first generate a policy network from the self-play results of a heuristic AI.

Our algorithm is evaluated by two sets of experiments, with existing Doudizhu AI's and human players respectively. We observe that our algorithm improved itself through selfplay, and made significant progress after days of training with modest computational power. It dominates all other Doudizhu AI's we are aware of, and shows a comparable strength against human experts. The playing style of our program looks more like human than other AIs.

The main contributions of this paper are:

- FPMCTS that enables policy iteration framework to work for multi-player imperfect-information games;

- Inference algorithm that takes advantage of players' policies directly;

- AlphaZero-like deep reinforcement learning framework that combines neural networks with FPMCTS and the inference algorithm in a self-play procedure;

- An expert-level Doudizhu AI via self-play.

\section{Related Works}

Perfect Information Monte Carlo (PIMC) is used by the classical bridge algorithms like GIB [Ginsberg, 1999]. Despite 
its success on imperfect information games [Long et al., 2010], PIMC still suffers from problems like Strategy Fusion and Non-Locality in reality.

MCTS is a popular search framework in perfect information games. In imperfect information games, the main challenge is that the asymmetry of information in an extensive form game makes constructing a single collective search tree problematic.

Several approaches are proposed to fix the problems caused by imperfect information. Information Set MCTS (ISMCTS) [Cowling et al., 2012] aimed to resolve the fusion problems. It grows a tree over information sets for each player instead of constructing a separate tree for each determinization. ISMCTS suffered from the information leaking problem, as pointed out by [Furtak and Buro, 2013]. [Heinrich and Silver, 2015] introduced Self-play MCTS and used a separate search tree for each player. The tree was grown on each player's own information state and was guaranteed to be a proper tree by perfect recall. Although each node in self-play MCTS contained an information set, the algorithm still had convergence problems. Smooth-UCT introduced a smoother action choosing scheme for better convergence property, but the algorithm might still need a lot of simulation steps to converge because of the iterative tree structure.

There are attempts to solve imperfect information games using deep reinforcement learning. [Heinrich et al., 2015] and [Heinrich and Silver, 2016] introduced Neural Fictitious Self-Play (NFSP), it calculated the best response for a given stationary policy and used a deep reinforcement learning approach to update that policy.

Doudizhu has not seen too many efficient solutions. [Powley et al., 2011] observed that games like Doudizhu with large branching factor were difficult for ISMCTS.

Our work combined the thoughts of self-play MCTS and NFSP. We proposed FPMCTS, a variant of MCTS which uses a neural network to store the policy. The best responses were computed via FPMCTS and the neural networks were updated in an AlphaZero-like framework. We implemented this idea and got a strong Doudizhu AI.

\section{Background}

\subsection{Doudizhu}

Doudizhu (a.k.a. Fight the Landlord) is one of the most popular card games in China. There are more than 800 million registered users and 40 million daily active players on the Tencent mobile platform for Doudizhu. The game is in the genre of climbing and shedding, and is described as easy to learn but hard to master.

The standard version of Doudizhu is played by three players with a pack of 54 cards including two jokers, red and black. The cards rank from high to low: Red Joker(B), Black Joker(L) 2, A, K, Q, J, 10(T), 9, 8, 7, 6, 5, 4, 3. Suits are irrelevant. The game begins with each of the three players being dealt 17 cards, with the remaining 3 cards dealt face-down.

The play of the game consists of two phases: bidding and cardplay. The bidding phase, which is not considered in this paper, designates one of the players as the landlord, which is analogous to the declarer in bridge. Unlike bridge, there are no permanent alliances of players cross games, and the two players who lose the bidding become partners (i.e. peasants) for the current game. We use letters to denote the positions: $\mathbf{C}$ for the landlord, $\mathbf{D}$ and $\mathbf{E}$ for the peasant on the right-hand side and left-hand side of the landlord respectively.

Once the landlord picks up the three face-down cards, card play begins. The game is played in rounds, and the landlord starts the first round. In any round, the first player may play any legal combination (single, pair, chain, etc.). Each subsequent player must either pass or beat the previous hand by playing a higher combination of the same hand category. There are just two exceptions: a rocket (a pair of jokers) can beat any combination, and a bomb (four cards of the same rank) can beat any combination except a higher bomb or rocket. The round continues until two consecutive players pass. When one round ends, the person playing the last cards in current round leads the next round.

The landlord wins the game if she gets rid of all cards on hand first; the peasants win otherwise. Each bomb or rocket played during the card play phase doubles the score for everyone. More thorough introductions to Doudizhu are available online at http://www.pagat.com/climbing/doudizhu.html.

\subsection{Extensive-Form Games}

Extensive-form games are a model of sequential interaction involving multiple agents. The representation is based on a game tree and consists of the following components: $\mathcal{N}=$ $\{1,2, \ldots, n\}$ denotes the set of players. $\mathcal{S}$ is a set of states corresponding to nodes in a finite rooted game tree. For each state node $s \in \mathcal{S}$ the edges to its successor states define a set of actions $\mathcal{A}(s)$ available to a player in state $s$. The player function $\rho: \mathcal{S} \rightarrow \mathcal{N}$ determines who is to act at a given state. For each player $i$ who cannot observe the full state, there is corresponding set of information states $\mathcal{U}^{i}$ and an information function $\mathcal{I}^{i}: \mathcal{S} \rightarrow \mathcal{U}^{i}$ that determines which states are indistinguishable for the player by mapping them on the same information set $u \in \mathcal{U}^{i}$. Finally, $R: \mathcal{S} \rightarrow \mathbb{R}^{n}$ maps terminal states to a vector whose components correspond to each player's payoff. The reward vector sums to zero for zerosum games. A policy $\pi^{i}$ for player $i$ maps each state $s$ with $\rho(s)=i$ to a probability distribution over $\mathcal{A}(s)$.

For Doudizhu, each player can only observe his own cards $c_{j}^{i}$ and action history $H_{j}=a_{0}, a_{1}, \ldots, a_{j}$ at time $j$. Therefore, the decision-making process in $u^{i}$ is a single agent MDP since other players' actions can be treated as feedback from the environment.

\section{Extending AlphaZero to Multi-Player Imperfect-Information Game}

In this section we describe the components introduced to make the AlphaZero-like training possible. The first component is FPMCTS, which is a variation of MCTS in an imperfect information game. The second component is policyvalue networks that serve as opponents strategies. The third component is inference of other players' cards. Last but not least, we describe how to adjust self-play in our case. 


\subsection{Fictitious Play MCTS}

The computational complexity brought by updating other players' choice recursively [Heinrich and Silver, 2015] motivated us to grow a separate search tree for each player. The fact that human players might choose pre-determined over rational actions [Ponsen et al., 2010] made us believe we could use policy network to approximate opponent responses. We call our algorithm Fictitious Play MCTS (FPMCTS). The term fictitious play [Brown, 1951] stands for our assumption that other players would always adhere to static but powerful strategies. We designed FPMCTS to make AlphaZero work for Doudizhu. [Ponsen et al., 2010] proposed a similar idea in the setting of Poker. These two approaches were developed independently for different purposes. Despite their similarities at the top-level, the two approaches have some differences: [Ponsen et al., 2010] used traditional MCTS with rollout and UCT. We use a value network to approximate the value of the leaf node, and p-UCT with a prior probability computed from a neural network for each action.

In the search tree for a specific player, there are two types of nodes: The decision nodes are the ones where the current player acts, and the rest are chance nodes. We merge the two opponents' consecutive actions into one chance node. In the section below, we use $i$ to denote the current player.

At decision nodes, we use p-UCT to select actions:

$$
\pi_{\text {tree }}\left(u^{i}\right)=\underset{a \in \mathcal{A}\left(u^{i}\right)}{\arg \max }\left[Q\left(u^{i}, a\right)+\pi^{i}\left(u^{i}, a\right) \frac{\sqrt{N\left(u^{i}\right)}}{1+N\left(u^{i}, a\right)}\right]
$$

where $Q\left(u^{i}, a\right)$ is the average Q-value by choosing action $a, N\left(u^{i}\right)$ is the number of times $u^{i}$ has been visited, and $N\left(u^{i}, a\right)$ is the number of times $a$ has been taken. Values of decision nodes are estimated by a function $v^{j}$. To avoid the non-locality problem, we choose several deterministic worlds at the root node before the search begins by an inference algorithm, which we will describe in Section 4.3. During the expansion phase, traditional MCTS expands a node by one level, and FPMCTS expands a node by two levels so that new leaf nodes are decision nodes unless the game ends at a chance node at next level.

At chance nodes, other players' moves are sampled from their policies. The network is updated after the self-play episode ends.

\subsection{Policy-Value Network}

Doudizhu has some specific hand categories that can make the action space very large. One example is Trio with single card, where any trio can be combined with an individual card as kicker, e.g.: 3334,3335 . This combination with kicker leads combinatorial explosion of the action space. To reduce the action space size, we only encode rank and category in the policy output. After the policy network produces answers, we use a kicker network and several heuristic rules to convert policy outputs to actual moves.

Since the neighboring relations between different card ranks are quite crucial in Doudizhu, we use 1D-convolutional layers. The network $f_{\theta}(s)=(\mathbf{p}, v)$ contains 10 residual blocks with batch normalization and rectifier non-linearities. The parameters of $f_{\theta}$ are adjusted by the gradient of a loss

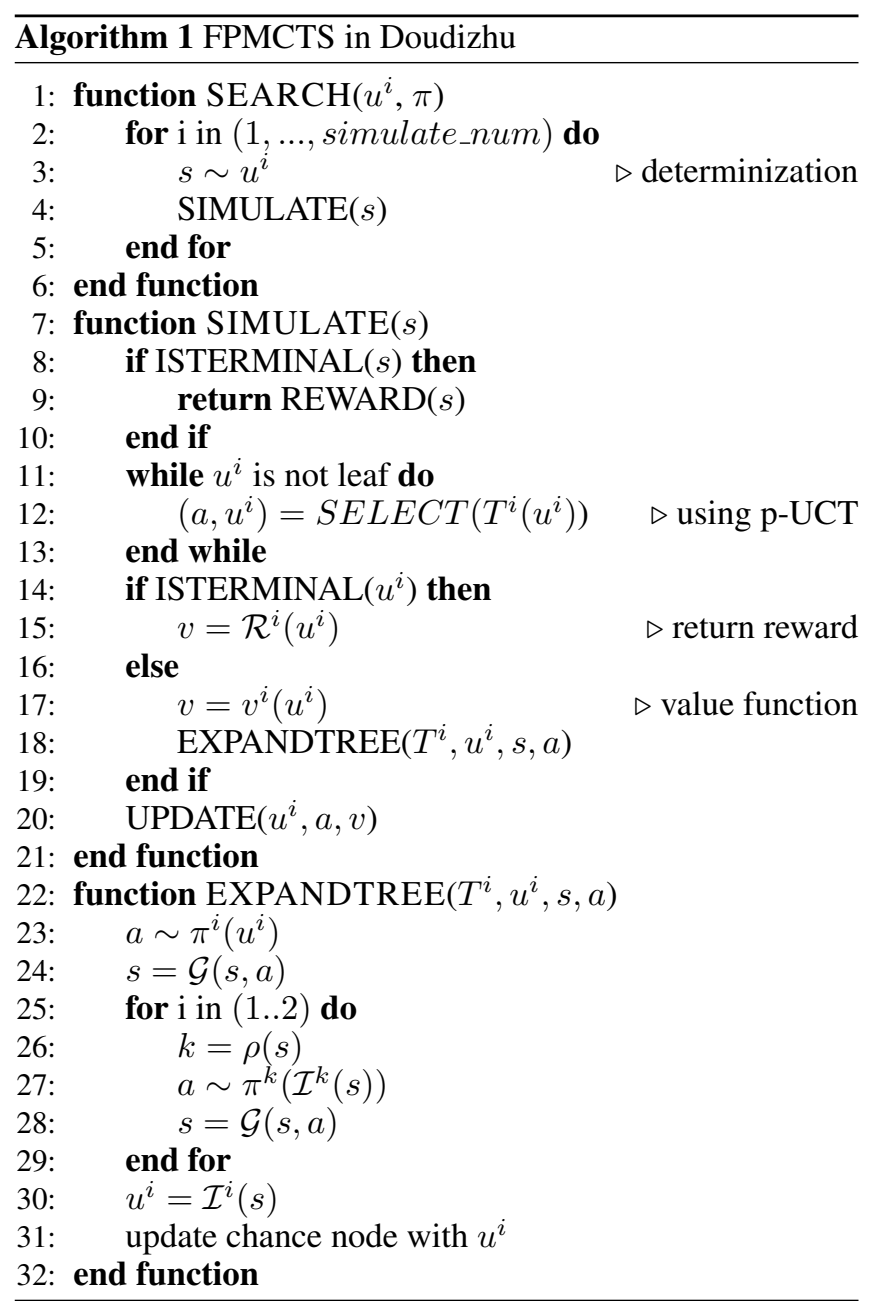

function that sums the over mean-squared error and crossentropy losses.

$$
l=C_{1}(z-v)_{2}-\pi^{\top} \log \mathbf{p}+C_{2}\|\theta\|_{2}
$$

where $C_{1}$ is used to rescale the value error since $v$ is normalized, and $C_{2}$ is a parameter controlling the level of $L_{2}$ weight regularization.

The input of the network is a condensed $15 \times 7$ matrix of current information state, including current player's cards, union of other players' cards, recent three actions, and union of played cards. The output is a matrix of legal moves classified into $n$ separate buckets according to the hand categories.

Our input and output encoding can be viewed as a tradeoff between precision and speed. In practice, the encoding has dramatically improved the training efficiency with few issues.

\subsection{Inference}

Players involved in imperfect information games can only observe a portion of the world, so making a good inference about the unseen portion can usually result in better action selections. A straightforward way to generate a deterministric world is random selection, but it fails to incorporate valuable knowledge from current game history. Therefore, 


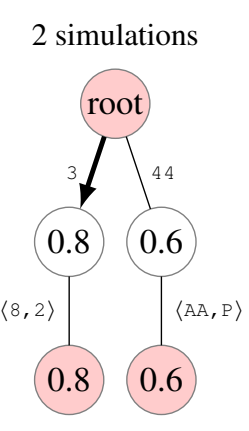

Peasnant D's round:

My(D's) Cards: LJJ 555443

Landlord C's cards count: 9

Peasant E's cards count: 3

$\mathrm{C}$ and E's cards: B2AAK9988833

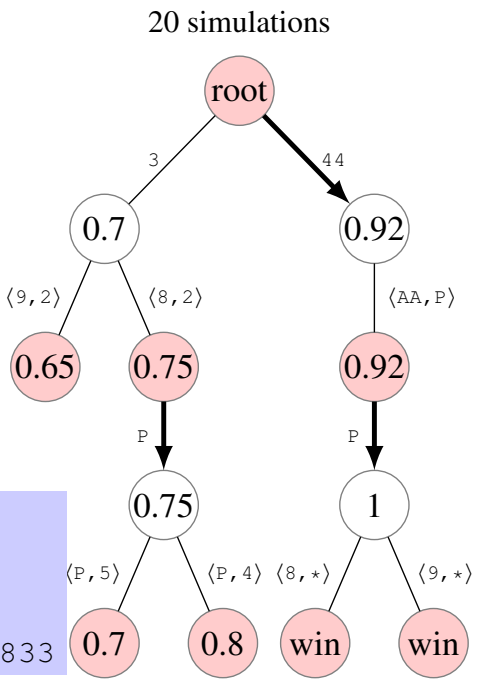

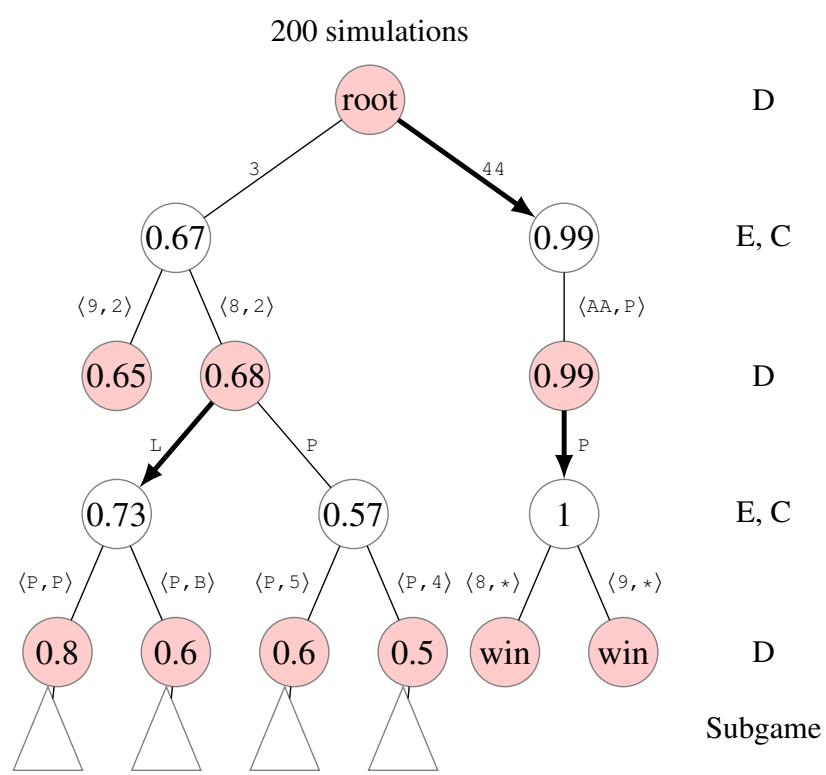

Figure 1: DeltaDou's search algorithm. This graph shows the D's initial choice 3 was corrected by the FPMCTS to $\mathbf{4 4}$, a cooperating approach with his teammate E. This action led E whose cards was inferred as AA 8 or AA 9 with $99 \%$ confidence got rid of all his cards first, and thus, peasants won the game. Each node would be an information set, storing its value and visit number. Edges from hollowed nodes mean explored choices of D , and arrows stand for D's best choices in corresponding nodes. Within each iteration, responses from other players would be determined by the inference and policy, and each node would be updated to track the average value of all evaluations $r(*)$ and $v_{\theta}(*)$ in expanded subtrees below it

researchers have designed several ways to bias the cards' distribution. Ponsen [Ponsen et al., 2010] has shown that the Bayesian Method equipped with a dependable policy regression model would be quite effective in Poker Games. Similarly, in Doudizhu, we take the output of policy-value network as a policy approximator to improve our inference.

Theoretically, the probability distribution for the real world could be computed precisely by the Bayesian Formula. We use $\mathcal{S}$ to denote all possible worlds of a specific information state. For any determinized situation $s \in \mathcal{S}$, its prior probability could be denoted as $p(s)$, and its history could be denoted by sequence $h(\mathcal{S})=h(s)=a_{1}, a_{2}, \cdots, a_{k}$. The policy could be denoted as $\operatorname{Pr}(a \mid s)=\operatorname{Pr}\left(a \mid \mathcal{I}^{\rho(s)}(s)\right)$. History situations $\left\{s_{1}, s_{2}, \cdots, s_{k}\right\}$ of this current situation $s$ would be derived by adding the played cards back. Therefore, the posterior probability $p(s \mid H)$ of the situation $s$ in the view of the current player could be computed as follows.

$$
p(s \mid H)=\frac{p(s) p(H \mid s)}{\Sigma_{s^{\prime} \in S} p\left(s^{\prime}\right) p\left(H \mid s^{\prime}\right)}
$$

in which for any $H=a_{1}, a_{2}, \cdots, a_{k}$,

$$
p\left(a_{1}, a_{2}, \cdots, a_{k} \mid s\right)=\prod_{s_{j}, \rho\left(s_{j}\right) \neq x} \operatorname{Pr}\left(a_{j} \mid s_{j}\right)
$$

However, in practice, there still exist several challenges [Buro et al., 2009]. First, calculating $p(s \mid H=h(s))$ for a large number of possible worlds is intractable. Second, a tight policy would make the prediction brittle in the face of players who do not play like us. Therefore, we designed a two-phase inference algorithm which can offer credible sampled worlds using modest hardware resources.
In Phase 1, a large number of deterministric worlds are randomly generated, then a small portion of the generated worlds would be retained based on filter scores. The scores are given by a 6-layer 1D-convolutional neural network trained from self-play games.

In Phase 2, the Bayesian method and the policy-value network are used to compute approximate posterior probabilities of samples generated in Phase 1. In detail, the policy $\operatorname{Pr}(a \mid s)$ for a certain $s$ could be derived from $f_{\theta}(s)=(\mathbf{p}(s), v)$ by applying a temperature operator. The temperature operator is used to make the inference algorithm more robust when the human opponents have different play styles.

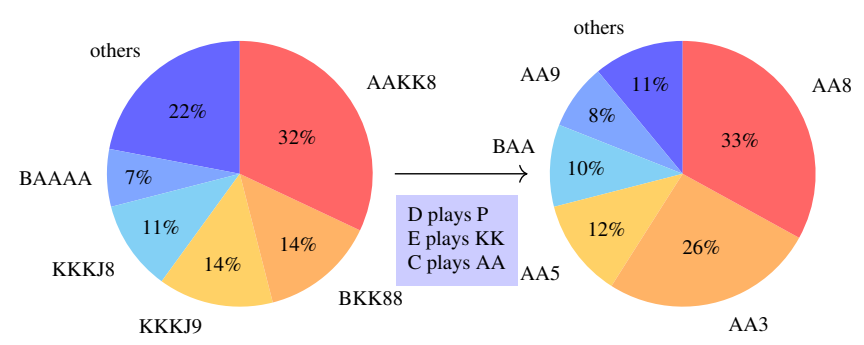

Figure 2: Inference example. This scenario shows the change of peasant D's guess on peasant E's cards. After a round of play, the probability of peasant E having exactly two A's increased from $40 \%$ to $93 \%$ from the point of view of $D$. 


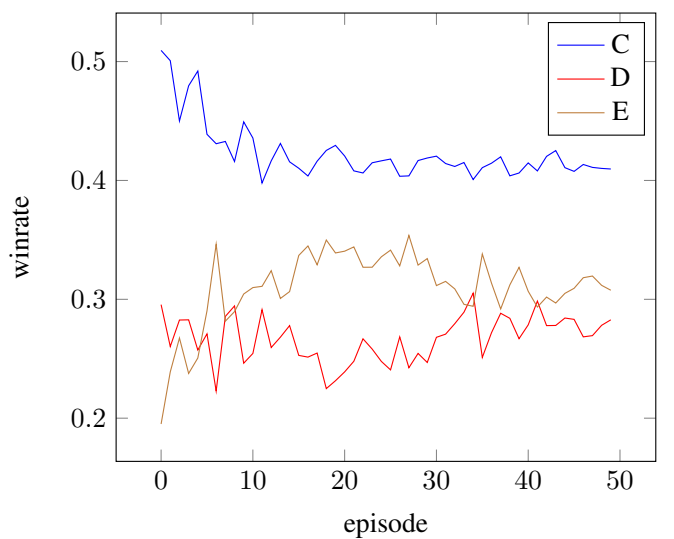

Figure 3: WP of DeltaDou each position in self-play games. WP of $\mathrm{C}$ is up to $50 \%$ in early period of self-play, while $\mathrm{D}$ wins more than E. As D learns how to let $\mathrm{E}$ wins by send him the hand he wants, Winning rate of E goes up and up to $35 \%$ in around episode 20 . The tactic of $\mathrm{E}$ to use an appropriate move to let $\mathrm{D}$ wins is learned later and D's WP goes up while D and E forms a more stronger peasants pair.

\subsection{Self-play}

Self-play is the key process to improve the performance of DeltaDou. Our procedure is similiar to that of AlphaZero except that we maintain one network for each positions $(\mathbf{C}, \mathbf{D}$, and $\mathbf{E}$ ) respectively. We use randomly generated deck in each game for self-play. During self-play, We find that DeltaDou struggles to play correctly in some rare cases.

Strategies for each state during self-play and game results are stored in a reservoir that is used to train the policy-value network for each player. The algorithm then updates itself iteratively as what is done in AlphaZero.

\section{Experiments}

In this section, we first describe how to train the policy-value network in DeltaDou. Then we show that DeltaDou is much stronger than other existing programs and appears to have reached human expert strength using performance results.

\subsection{Training Process}

We used a hand-coded heuristic algorithm to bootstrap the training process. In the first phase, 200,000 games were selfplayed by the heuristic algorithm, then the game results were used to generate the initial policy-value network under supervised learning.

During self-play, each episode contains 8000 games, and FPMCTS contains 400 playouts. Inference is used when any player has fewer than 15 cards in hand. At the beginning of the self-play process, a high temperature is used to reduce the overfitting problem of policy, and the temperature cools down during self-play.

In order to check the progress of the self-play, we need to evaluate the performance of the agent. Our approach is inspired by teams tournament for duplicate bridge. When two teams A and B are compared with each other, the same hand is played twice: First, Team A plays as the landlord and Team

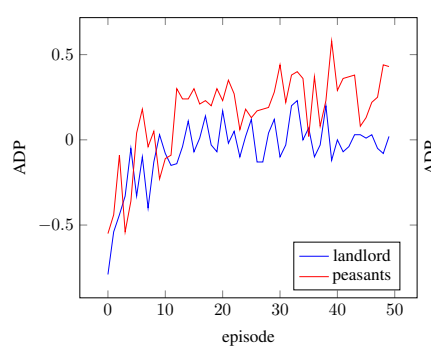

(a) ADP by position

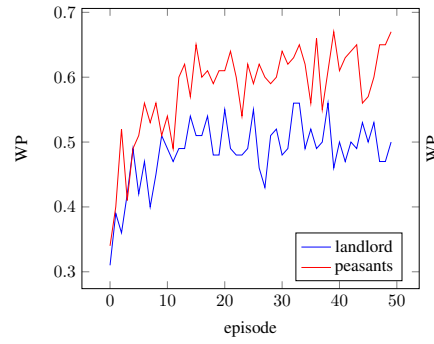

(c) WP by position

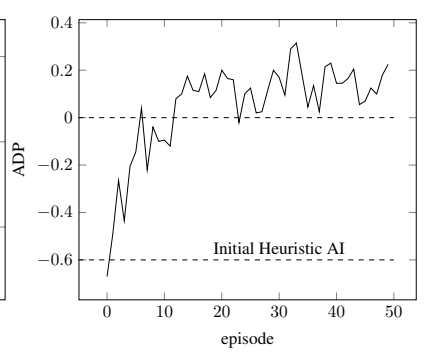

(b) overall ADP

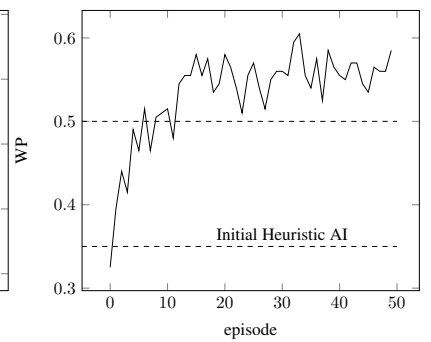

(d) overall WP
Figure 4: Learning curve of the self-play training. The testing data set consists of a fixed set of 100 games. The benchmark program is a strong heuristic algorithm XDou. We also mark the performance of the heuristic algorithm that was used to generate the initial neural network for self-play. DeltaDou beated the initial heuristic AI and XDou after 3 and 10 episodes respectively. Also note that the advantage of DeltaDou against XDou is mainly from the peasants side.

$\mathrm{B}$ as the peasants, and then Team A plays as the peasants and Team B as the landlord. The following two metrics are used to compare the performances of the teams:

- ADP (Average Difference in Points): The difference of the points scored per game of the two teams for the given hand set. For each game, the winning team gets 1 point and the losing team gets -1 point. Every bomb played during the game doubles the score.

- WP (Winning Percentage): The fraction of games a team won, i.e. number of games won divided by number of games played. A team has a better performance than the opponent team if the former got a WP higher than $50 \%$.

The performance gain of the learning process was measured against XDou, our strongest heuristic program. The learning curve of DeltaDou is shown in Figure 4.

\subsection{Comparison to Doudizhu Baseline}

We launched a tournament that include the two opponent side each play at landlord position and peasants position for 100 decks. WP and ADP will be used as playing strength metrics.

We are not aware of any AI competition where strong Doudizhu AI's can compete with each other. Therefore, we chose two Doudizhu solvers to benchmark DeltaDou. The fisrt one is RHCP, an open source heuristics-based algorithm available online (https://blog.csdn.net/sm9sun/article/details/ 70787814). It decides the hand to play purely by some handcrafted hand value estimation function. The second one is 
Proceedings of the Twenty-Eighth International Joint Conference on Artificial Intelligence (IJCAI-19)

\begin{tabular}{rcc}
\hline & WP & ADP \\
\hline XDou vs RHCP & $79 \%$ & +0.88 \\
DeltaDou vs RHCP & $75 \%$ & +0.72 \\
DeltaDou vs XDou & $59 \%$ & +0.28 \\
\hline
\end{tabular}

Table 1: Head-to-head tournament results between DeltaDou and baseline programs. DeltaDou and XDou are both much stronger than RHCP. In fact, XDou performed even better by exploiting the weak opponent more. However, DeltaDou showed statistically significant margin over XDou when they faced each other directly.

\begin{tabular}{lcclcc}
\hline & \multicolumn{2}{c}{ RHCP } & & \multicolumn{2}{c}{ XDou } \\
\cline { 2 - 3 } \cline { 5 - 6 } & WP & ADP & & WP & ADP \\
\hline Policy & $58 \%$ & +0.19 & & $49 \%$ & -0.08 \\
DeltaDou & $75 \%$ & +0.72 & & $59 \%$ & +0.28 \\
\hline
\end{tabular}

Table 2: DeltaDou vs Policy against baseline. The policy algorithm achieves comparable performance as XDou, and it is still left far behind by DeltaDou, which used both looking forward search and inference.

XDou, a strong Doudizhu AI program developed by our team. It integrates sophisticated heuristics and a PIMC-based endgame solver.

The version of DeltaDou used for the evaluation contains a neural network trained for 80 episodes (i.e. 640,000 games). It took 2 months to train the network on 68 CPUs. The number of simulations in MCTS is set to 600 and c-pUCT is set to 2 .

As neural network computation is required by both looking forward search and inference, DeltaDou is a quite heavy method. Extracting the policy from the neural network greedily resulted in a pretty fast solution. We measured this algorithm against our base line programs. Table 2 shows that the pure policy algorithm is comparable with XDou, and is dominated by DeltaDou as expected.

The agent with inference got WP 54.5\% and ADP +0.145 in the heads-up game against the agent without inference. The result shows that our inference algorithm is quite effective.

\subsection{Comparison to Human Player}

Several winners of online Doudizhu tournaments were teamed up to play with DeltaDou. The experiments with human players is sort of time-consuming, especially when the human team plays on the peasant side. Both DeltaDou and the human team played the same 100 games on landlord and peasant side. Human players need to remember what cards has been played, since there is no hint about what cards are not shown yet from the user interface of the game. There is no timing constraints for playing a hand. The DeltaDou used for this experiment is the same version as in the experiment against other AI's. It was ran on a single 8-core computer with the average time for a move of roughly 5 to 8 seconds.

When a team won 5 more games out of 100 games against the opponent, we treat them as teams at different skill levels. DeltaDou won 3 less games out of the 100 games to the human team, and has achieved comparable performance as our

\begin{tabular}{ccc}
\hline & WP (landlord) & ADP (landlord) \\
\hline D-D & $39 \%$ & -0.44 \\
D-H & $33 \%$ & -0.61 \\
H-D & $36 \%$ & -0.47 \\
\hline
\end{tabular}

(a) Results of different position combinations

\begin{tabular}{ccc}
\hline & WP & ADP \\
\hline DeltaDou vs Human & $48.5 \%$ & -0.07 \\
\hline
\end{tabular}

(b) Overall performance

Table 3: DeltaDou's tournament results vs human team. X-Y means $\mathrm{X}$ plays as the landlord and Y plays as the peasants. D and $\mathrm{H}$ denotes DeltaDou and the human team respectively. DeltaDou is stronger than human as the landlord ( $39 \%$ vs $36 \%,-0.44$ vs -0.47 ) and is weaker than human as the peasants $(61 \%$ vs $67 \%, 0.44$ vs 0.61$)$. The overall performance of DeltaDou is slightly worse than the human opponents, with $48.5 \%$ and -0.07 for WP and ADP respectively.

human experts. A drawback of DeltaDou is that sometimes it misses a straight win that is obvious for the human players. On the other side, some nice play by DeltaDou are very impressive. Most of the time, these plays require precise analysis and calculation, and are difficult to be found by human experts.

\section{Conclusion and Future Work}

In this paper, we present our Doudizhu AI, DeltaDou, which dominates existing Doudizhu programs and plays roughly at human expert level. DeltaDou successfully integrates FPMCTS and inference algorithm into an AlphaZero-like framework. As far as we know, it is the first reinforcement learning algorithm that reaches expert level in a difficult multi-player imperfect information game with limited hardware resource and training time.

In future work, we would like to remove the heuristicbased neural network for bootstrapping so that the training process can start from scratch without any human knowledge. We have started experimenting with opponent modeling, which may help the AI to reach super-human level strength by exploiting the weakness of opponents more and by better understanding its partner. Finally, we will try to adapt our framework to other imperfect information card games such as bridge or skat.

\section{Acknowledgments}

The authors would like to thank the anonymous reviewers for their valuable comments. Our colleagues, Guangxin Ren, Chang $\mathrm{Wu}$, and $\mathrm{Wen} \mathrm{Xu}$, shared their know-how on the Doudizhu AI and contributed code libraries and other useful tools. 


\section{References}

[Brown and Sandholm, 2018] Noam Brown and Tuomas Sandholm. Superhuman AI for heads-up no-limit poker: Libratus beats top professionals. Science, 359(6374):418424, 2018.

[Brown, 1951] George W Brown. Iterative solution of games by fictitious play. Activity analysis of production and allocation, 13(1):374-376, 1951.

[Buro et al., 2009] Michael Buro, Jeffrey Richard Long, Timothy Furtak, and Nathan R Sturtevant. Improving state evaluation, inference, and search in trick-based card games. In IJCAI, pages 1407-1413, 2009.

[Campbell et al., 2002] Murray Campbell, A Joseph Hoane Jr, and Feng-hsiung Hsu. Deep blue. Artificial intelligence, 134(1-2):57-83, 2002.

[Cowling et al., 2012] Peter I Cowling, Edward J Powley, and Daniel Whitehouse. Information set monte carlo tree search. IEEE Transactions on Computational Intelligence and AI in Games, 4(2):120-143, 2012.

[Furtak and Buro, 2013] Timothy Furtak and Michael Buro. Recursive monte carlo search for imperfect information games. In Computational Intelligence in Games (CIG), 2013 IEEE Conference on, pages 1-8. IEEE, 2013.

[Ginsberg, 1999] Matthew L Ginsberg. Gib: Steps toward an expert-level bridge-playing program. In IJCAI, pages 584-593. Citeseer, 1999.

[Heinrich and Silver, 2015] Johannes Heinrich and David Silver. Smooth uct search in computer poker. In IJCAI, pages 554-560, 2015.

[Heinrich and Silver, 2016] Johannes Heinrich and David Silver. Deep reinforcement learning from selfplay in imperfect-information games. arXiv preprint arXiv:1603.01121, 2016.

[Heinrich et al., 2015] Johannes Heinrich, Marc Lanctot, and David Silver. Fictitious self-play in extensive-form games. In International Conference on Machine Learning, pages 805-813, 2015.

[Long et al., 2010] Jeffrey Richard Long, Nathan R Sturtevant, Michael Buro, and Timothy Furtak. Understanding the success of perfect information monte carlo sampling in game tree search. In Twenty-Fourth AAAI Conference on Artificial Intelligence, 2010.

[Moravčík et al., 2017] Matej Moravčík, Martin Schmid, Neil Burch, Viliam Lisỳ, Dustin Morrill, Nolan Bard, Trevor Davis, Kevin Waugh, Michael Johanson, and Michael Bowling. Deepstack: Expert-level artificial intelligence in heads-up no-limit poker. Science, 356(6337):508-513, 2017.

[Ponsen et al., 2010] Marc Ponsen, Geert Gerritsen, and Guillaume Chaslot. Integrating opponent models with monte-carlo tree search in poker. In Workshops at the Twenty-Fourth AAAI Conference on Artificial Intelligence, 2010.
[Powley et al., 2011] Edward J Powley, Daniel Whitehouse, and Peter I Cowling. Determinization in monte-carlo tree search for the card game dou di zhu. Proc. Artif. Intell. Simul. Behav, pages 17-24, 2011.

[Schaeffer et al., 2007] Jonathan Schaeffer, Neil Burch, Yngvi Björnsson, Akihiro Kishimoto, Martin Müller, Robert Lake, Paul Lu, and Steve Sutphen. Checkers is solved. science, 317(5844):1518-1522, 2007.

[Silver et al., 2016] David Silver, Aja Huang, Chris J Maddison, Arthur Guez, Laurent Sifre, George Van Den Driessche, Julian Schrittwieser, Ioannis Antonoglou, Veda Panneershelvam, Marc Lanctot, et al. Mastering the game of go with deep neural networks and tree search. nature, 529(7587):484, 2016.

[Silver et al., 2018] David Silver, Thomas Hubert, Julian Schrittwieser, Ioannis Antonoglou, Matthew Lai, Arthur Guez, Marc Lanctot, Laurent Sifre, Dharshan Kumaran, Thore Graepel, et al. A general reinforcement learning algorithm that masters chess, shogi, and go through selfplay. Science, 362(6419):1140-1144, 2018.

[Tesauro, 1995] Gerald Tesauro. Td-gammon: A selfteaching backgammon program. In Applications of Neural Networks, pages 267-285. Springer, 1995. 